\title{
BLEND ELECTROSPINNING OF POLY(E-CAPROLACTONE) AND POLY(ETHYLENE GLYCOL-400) NANOFIBERS LOADED WITH IBUPROFEN AS A POTENTIAL DRUG DELIVERY SYSTEM FOR WOUND DRESSINGS
}

\author{
Tabinda Riaz ${ }^{1,2,3}$ Nabyl Khenoussi ${ }^{2}$, Delia Mihaela Rata ${ }^{4}$, Leonard Ionut Atanase ${ }^{4}$, \\ Dominique C. Adolphe ${ }^{2, *}$, Christelle Delaite ${ }^{1}$
1 Laboratoire de Photochimie et d'Ingenierie Macromoleculaires, 3-bis rue Alfred Werner, Université de Haute Alsace, 68093 Mulhouse, France 2 Laboratoire de Physique et Mecanique Textiles, 11 rue Alfred Werner, Université de Haute Alsace, 68093 Mulhouse, France 3 Department of Textile Engineering and Technology, University of the Punjab, 54590 Lahore, Pakistan 4 Faculty of Dental Medicine, Str. Pacurari Nr.11, University "Apollonia", 700511 lasi, Romania ${ }^{*}$ Corresponding author. E-mail: dominique.adolphe@uha.fr

\begin{abstract}
:
Electrospinning (ES) is a versatile and diverse technique to fabricate nano and micro fibers that could be utilized as drug delivery systems. The aim of this research was the fabrication and characterization of drug loaded nanofibrous scaffold produced by single-needle ES using poly(E-caprolactone) (PCL) and poly(ethylene glycol-400) (PEG) and to investigate the potential of this material as a drug delivery system. A model drug, Ibuprofen (IBU), was used. Ibuprofen is a medicine that is a non-steroidal, anti-inflammatory drug (NSAID). Two concentrations of IBU, 5 wt\% and 7 wt\%, were incorporated for the ES of PCL and PCL/PEG nanofibers. Characterization of nanofibers was done by using Scanning Electron Microscopy (SEM), Differential Scanning Calorimeter (DSC), Thermogravimetric Analysis (TGA), and Water Contact Angle Measurements. The impact of IBU on nanofibers' properties such as morphology, diameters, hydrophilicity, and tensile strength was investigated. Finally, the drug release kinetics of IBU from nanofibers was analyzed and their percentage release efficiency of IBU (RE\%) was determined by UV-vis spectroscopy during $24 \mathrm{~h}$.
\end{abstract}

\section{Keywords:}

Poly(ethylene glycol)-400, blend electrospinning, crystallinity, drug-delivery system, Ibuprofen

\section{Introduction}

Wound healing is a complex tissue regeneration process that the human body undergoes to anticipate the affected area with ruptured cellular tissues due to any kind of injury. Inflammation in wounds is another problem that could turn a simple injury into a critical wound, especially in the cases of patients with diabetes or low immunity. In such cases, the curing process offers three main challenges: (a) to absorb the sepsis from wound, (b) to relieve the pain, and (c) to protect the wound from the external environment. For rapid healing, a wound is typically covered with a sterile dressing material to prevent infection and to facilitate fast recovery. Usually, antiseptic gels and creams loaded with drug of only $5 \mathrm{wt} \%$ at maximum concentration are applied to the wound as a first aid by paramedics [1]. It is required to change the dressing at regular intervals, preceded by wound cleaning that could be painful and exhausting at times for the patient. Low efficacy of such drug-induced ointments and frequent wound manipulations can often be costly and labor-intensive. Thus, the need for an advanced and multifunctional wound dressing is generated, which should exhibit controlled drug delivery and sustainable properties, such as good mechanical strength, hydrophilicity, and biodegradability [2-4].

In recent years, nanotechnology and nanofibers have gained significant attention in the field of biomedical science due to their amazing properties, such as very small size to volume ratio, high tenacity, and remarkable drug loading and release efficiencies. Various advanced fibers were invented that are biocompatible and biodegradable. These fibers were usually incorporated with nanoparticles and nanocapsules that were loaded with the targeted drug or by incorporating the drug directly to the polymer solution to fabricate multifunctional wound dressings. These innovations enabled mankind to effectively deal with special types of wounds, critical locations, and conditions of wounds [5-8].

Electrospinning (ES) has emerged as a promising technology that can be used to create versatile fibrous structures for biomedical, drug-delivery, and filtration applications. This technique is simple, robust, and cost effective to produce drug-loaded fibers with diameters ranging from nanoscale to submicron depending upon the applied parameters [9-11]. The basic principal of ES is to create a potential difference between two surfaces, a needle, and a collecting surface by applying voltage that overcomes the surface tension of the polymer solution at the tip of the needle and stretches the jet of solution to form nanofibers. The product is a fiber network that resembles the texture of a nonwoven textile material with pores which help in absorption of the wound exudate and permit oxygen to reach the wound, releasing the drug to cure pain and protecting it from the external environment. Many synthetic and natural biopolymers were electrospun and modified to meet the 
diverse needs according to the specific biomedical applications $[12,13]$. Among these polymers, poly $(\mathcal{E}$-caprolactone) (PCL) is a linear aliphatic polyester which is biodegradable and biocompatible with low melting point, high resilience, and pliability. It is hydrophobic and insoluble in aqueous solution but dissolves in chloroform, acetone, tetrahydrofuran (THF), dichloromethane, and mixtures with ethanol or methanol [1416].

To enhance the hydrophilicity of PCL, blending with poly(ethylene glycol) (PEG) with different molar masses is reported by various scientists as a successful solution. However, the literature review showed that scientists had used high molar masses of PEG to modify the properties of PCL nanofibers [17-20]. No study was found which reported the use of PEG-400 for PCL blend ES and characterization of resulting nanofibers. Thus, in this study a very low molar mass of PEG (Mn-400) was employed for the stated purpose. The PCL/PEG nanofibers were produced by single-needle ES and characterization of these nanofibers was done by Scanning Electron Microscopy (SEM), Differential Scanning Calorimeter (DSC), Thermogravimetric Analysis (TGA), Water ContactAngle Measurements, and Tensile Strength Testing. As mentioned earlier, nanofibers can be used as drug delivery systems for wound healing. Thus, Ibuprofen (IBU) was incorporated into these nanofibers as a model drug. It is a non-steroidal, antiinflammatory drug (NSAID), mostly used in wound dressings to cure pain and inflammation [21, 22]. IBU was directly added to the polymer solution in two different concentrations to gauge the impact caused by these concentrations on nanofibers' morphology, nanofibers diameter, and their crystallinity ratios. To investigate the scope of these IBU-loaded PCL nanofibers as a potential wound dressing, the drug release kinetics of IBU were studied by UV-Vis spectroscopy using characteristic absorption bands of drug.

\section{Experimental}

\section{$\underline{2.1 \text { Materials }}$}

PCL $(\mathrm{Mw}=80,000 \mathrm{~g} / \mathrm{mol})$ and PEG $(\mathrm{Mw}=400 \mathrm{~g} / \mathrm{mol})$ were purchased from Sigma-Aldrich and were used as received. Chloroform and THF were purchased from Carlo Erba with purity $>99.0 \%$. Ethanol and Acetone by Honeywell with purity $>99.5 \%$ and o-xylene from Sigma-Aldrich with purity $98.0 \%$ were procured. IBU (>98\% (Mw (= $206.28 \mathrm{~g} / \mathrm{mol})$ was sourced by from Sigma Sigma-Aldrich.

\subsection{Methods}

A homemade setup of single-needle ES was used for this research work with a syringe pump to maintain a uniform flow rate of solution. A high voltage source $(0-30 \mathrm{kV})$ was connected to an enclosed poly (methyl methacrylate) chamber to supply a controlled electric field between needle tip and the grounded collector that was made of $20 \times 20 \mathrm{~cm}$ Teflon plate. All nanofibrous samples were collected on this grounded plate that was covered with aluminum foil, and the samples have been collected using random location on the aluminum foil to be representative of the nano-filaments' population. PCL solution was filled in a $20 \mathrm{~mL}$ plastic syringe attached to a needle with an inner diameter of $0.4 \mathrm{~mm}$ gauge. All experiments were carried out at room temperature $\left(25 \pm 2{ }^{\circ} \mathrm{C}\right)$ and relative humidity was kept constant at $35 \pm 4 \%$. A schematic diagram of single-needle ES setup is shown in Figure 1 [23].

\subsection{Preparation of ES solution}

A homogeneous solution of $10 \mathrm{wt} \%$ PCL was prepared by using a mix of solvents, composed of chloroform and ethanol in 88:12 wt/wt ratio, respectively. To obtain a clear solution of $\mathrm{PCL}$, the solution was magnetically stirred overnight at room temperature. 10 wt\% PEG-400, with respect to weight of PCL, was added to this clear solution and it was again stirred for 15 min with magnetic stirrer. Finally, the solution was left in an ultrasonic bath for $5 \mathrm{~min}$ to remove air bubbles. Similarly, $5 \mathrm{wt} \%$ and $7 \mathrm{wt} \% \mathrm{IBU}$ with respect to the weight of $\mathrm{PCL}$, were directly added to the clear PCL and PCL/PEG-10\% solutions respectively and were magnetically stirred for $10 \mathrm{~min}$ each. Later on, these solutions were left in an ultrasonic bath for $5 \mathrm{~min}$ to remove air bubbles.

\section{$\underline{2.4 \text { Viscosity measurements }}$}

Viscosity measurements were performed for PCL solutions in different solvents such as chloroform, THF, acetone, o-xylene, and a binary solvent system of chloroform:ethanol (88:12 wt/ wt). For this purpose, an Anton Paar MCR-302 plate-plate rheometer was employed. All measurements were taken at a shear rate of $100 / \mathrm{s}$ and at room temperature $\left(25^{\circ} \mathrm{C}\right)$. For each sample, 20 measuring points were taken and for each point the time-setting was $12 \mathrm{~s}$. All solutions were clear and homogeneous and viscosity was measured as a function of time.

\section{$\underline{2.5 \text { Preparation of PCL film }}$}

A PCL film was used as a reference to measure the water contact angle of PCL and PCL/PEG-10\% nanofibers. To prepare the film, the PCL solution was prepared by using a solvent mix of chloroform, namely ethanol (88:12 wt/wt), and was magnetically stirred overnight. This solution was utilized to cast a smooth film on a Teflon sheet by using a manual bar coater of $100 \mu \mathrm{m}$ thickness. After coating a thin film of PCL solution on the Teflon sheet, the sheet was placed in a drying oven for $3 \mathrm{~h}$ at $40^{\circ} \mathrm{C}$ to evaporate all the solvents. After complete drying, it was used to measure the water-contact angle.

\subsection{Scanning electron microscopy}

SEM-JSM model IT100, was used to take high resolution images of nanofibers. Prior to SEM, all samples were sputtercoated with gold. Three images for each type of samples were taken at $10 \mu \mathrm{m}$ scale. Diameters of nanofibers were measured by using ImageJ software, which was used to take bar measurements. Fifty measurements were made per sample to ensure accuracy. Mean diameters, standard deviations, and coefficients of variation (CV\%) for all samples were calculated 


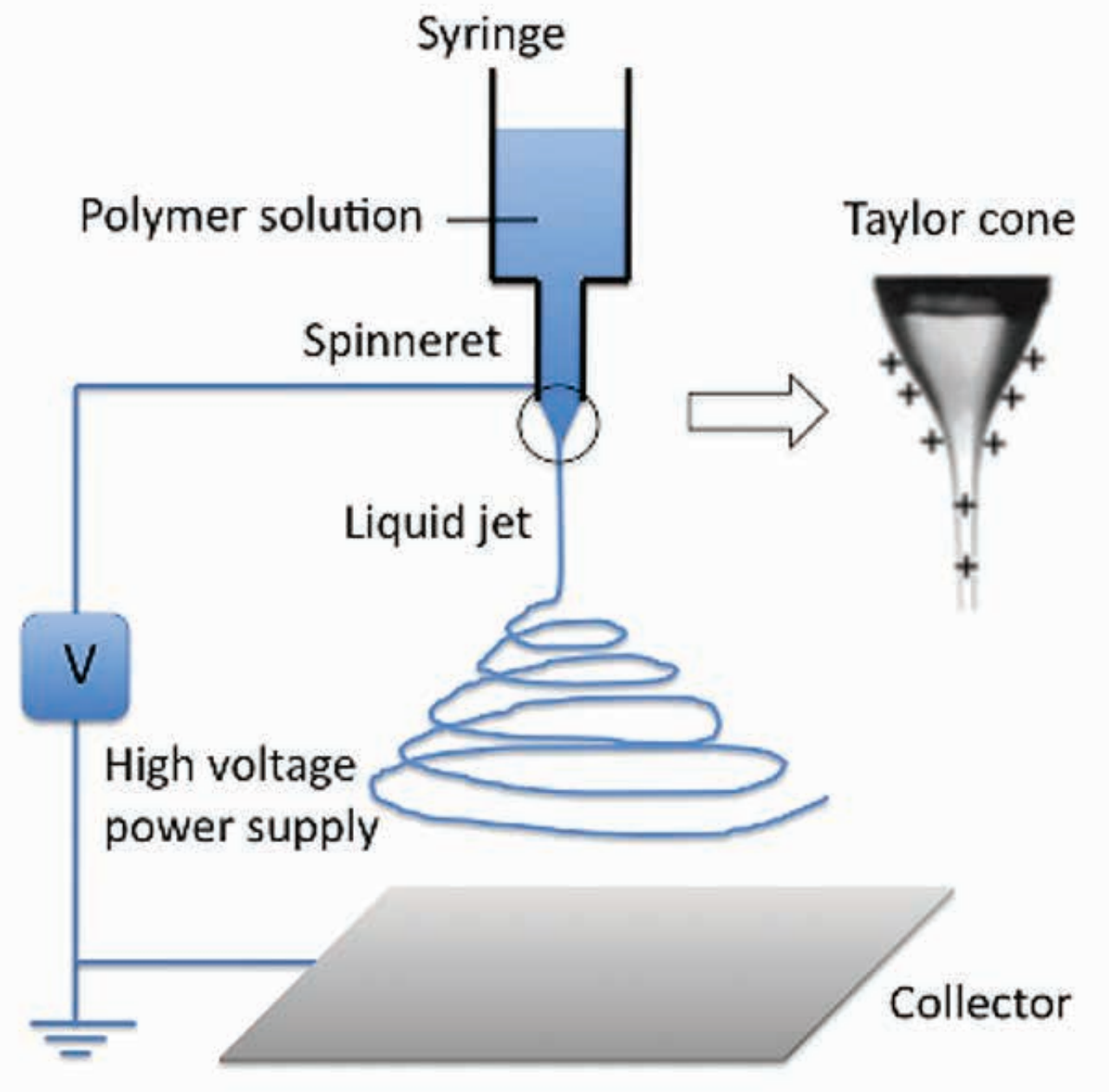

Figure 1. Schematic diagram of needle ES set-up [23]. ES, electrospinning.

to determine the overall morphology and homogeneity of nanofiber.

\section{$\underline{2.7}$ Contact angle measurement}

Water contact angle measurements were carried out by Drop Shape Analyzer (DSA 100 KRUSS GmbH, Germany) apparatus by Sessile drop method. A water droplet of $2 \mu \mathrm{L}$ was dispensed from the needle and was dropped on the samples placed underneath on a glass plate. The PCL film prepared on Teflon sheet was peeled off and then used for taking measurements. Distilled water was used as reference liquid and was allowed to drop automatically on the electrospun nanofibers. Contact angle measuring time was $10 \mathrm{~s}$ after the water droplet was dropped. Measurements were recorded by CCD video camera installed inside the instrument. Five droplets for each sample were deposited and analyzed.

\subsection{Differential scanning calorimetry}

Thermal properties of pure PCL, pure PEG, and all sets of $P C L$ and $P C L / P E G$ nanofibers were determined by using DSC model TA instrument Q200. Specimens of approximately $9 \mathrm{mg}$ in weight were sealed in non-hermetic aluminum capsules. Experiments were performed under nitrogen atmosphere with a single cycle of heating from $-80^{\circ} \mathrm{C}$ to $100^{\circ} \mathrm{C}$ at a rate of $10^{\circ} \mathrm{C} /$ min. The glass transition and melting temperatures of each sample were determined and the crystallinity ratios $(\mathrm{Xc})$ of $\mathrm{PCL}$ and PCL/PEG nanofibers were calculated. The values of $X_{c} \%$ of all nanofibers were calculated with reference to the content of PCL used for PCL/PEG blends. $X_{c} \%$ was determined by using the following Equation 1:

$X \mathrm{C}(\%)=\Delta \mathrm{H}_{\mathrm{m}} / \Delta \mathrm{H}_{\mathrm{m} \cdot \mathrm{PCL}} \times 100$

where $\Delta \mathrm{H}_{\mathrm{m}}$ is the melting enthalpy of PCL nanofibers and $\Delta \mathrm{H}_{\mathrm{m} . \mathrm{PCL}}$ is the melting enthalpy of $100 \%$ crystalline PCL, that is $142 \mathrm{~J} / \mathrm{g}$ [24].

\subsection{Thermogravimetric analysis}

To determine the thermal stability of nanofibers, TGA was performed by using TA instrument model Q500. All samples were analyzed under nitrogen with $10^{\circ} \mathrm{C} / \mathrm{min}$ rate of heating ramp till $800^{\circ} \mathrm{C}$.

\subsection{Uniaxial tensile strength analysis}

Rectangular strips of nanofibrous webs were cut from different areas of sample with a $20 \mathrm{~mm}$ gauge length and $5 \mathrm{~mm}$ width using a roller cutter. The values for thickness of each sample, at five different points, were measured using Schut's digital 
micrometer with a precision value up to $0.001 \mathrm{~mm}$. All the specimens were weighed on a micro balance. Different areas were chosen to cover all possible thicknesses of nanofibers. The specimens were conditioned in atmospheric conditions at $20 \pm 2^{\circ} \mathrm{C}$ and $65 \pm 2 \%$ relative humidity for $48 \mathrm{~h}$ before testing. Double-edge duct tape was placed on both edges of the sample to facilitate its grip between the jaws of tensile tester. Cut samples were sandwiched between two cardboard layers, which functioned as templates and made it possible to easily handle and grip the specimens in jaws for mechanical testing, as shown in Figure 2. The edges of the cardboard along with the specimen ends were placed between the grips of a tensile testing machine, thereby ensuring that the area of nanofibrous webs between the two jaws remained at $10 \mathrm{~mm}$. Tensile test was performed on MTS M/20 tensile testing machine using a load cell with a capacity of $10 \mathrm{~N}$ and an elongation rate of $10 \mathrm{~mm} / \mathrm{min}$. The cardboard templates were cut after fixing specimens between pneumatic clamps of the tensile machine and tests were performed at room temperature. Four samples for each specimen were tested to analyze the tensile strength.

\subsection{Drug Release Kinetics}

The in-vitro drug-release profile of IBU-loaded nanofibers was studied by using dialysis method. Each sample was introduced into a dialysis membrane and was suspended into $10 \mathrm{~mL}$ of buffer solution (phosphate buffer solution with $\mathrm{pH}$ 7.4) at $37 \pm 0.5^{\circ} \mathrm{C}$ under constant stirring. Certain volumes of solution (approximately $2 \mu \mathrm{L}$ ) were taken out at pre-established time intervals and the concentration was determined by UV-vis spectroscopy according to a calibration curve, which is shown

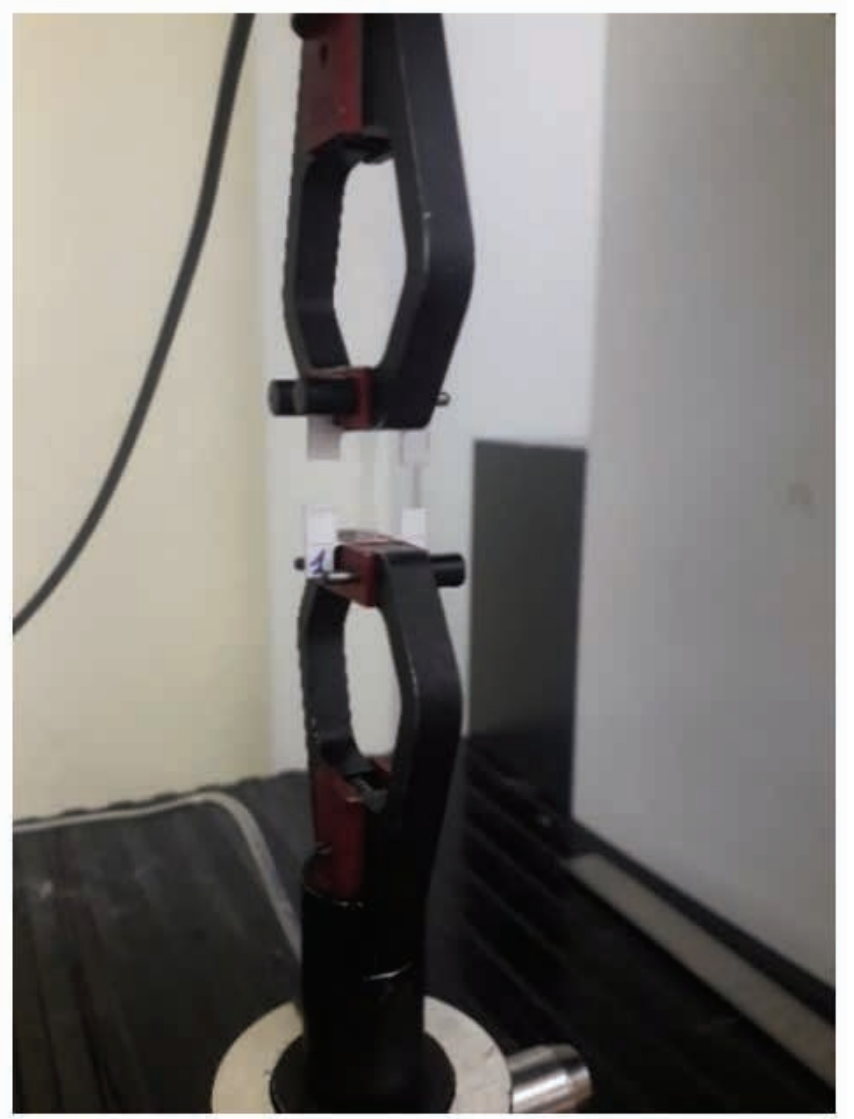

Figure 2. Specimen between the jaws of tensile strength tester. in Figure 3. The percentage release efficiency of IBU (RE\%) was calculated using the following Equation 2:

$\mathrm{RE} \%=\mathrm{mr} / \mathrm{ml} \times 100$

where $\mathrm{mr}$ is the amount of released IBU $(\mathrm{mg})$ and $\mathrm{mL}$ is the amount of loaded IBU $(\mathrm{mg})$ in nanofibrous webs.

\section{Results and discussion}

\section{$\underline{3.1 \text { Viscosity analysis of PCL solutions }}$}

$10 \mathrm{wt} \%$ concentration of PCL was taken to prepare the solutions with each solvent i.e., chloroform, chloroform:ethanol (88:12) $\mathrm{wt} / \mathrm{wt}, \mathrm{THF}$, acetone, and o-xylene. The viscosity values of $\mathrm{PCL}$ in chloroform and chloroform:ethanol (88:12 wt/wt) solvents were the highest in a range of $3,200-3,600 \mathrm{MPa} / \mathrm{s}$. On the other hand, the PCL solutions in THF, acetone, and o-xylene exhibited a different behavior with very low viscosity values of 600,500 , and $300 \mathrm{MPa} / \mathrm{s}$ respectively. It is established from the literature that viscosities that are excessively high or low are not favorable for the production of continuous and bead-free nanofibers [24, 25]. This vast difference in viscosities indicated the interaction among PCL molecular chains with different solvents. Higher viscosity value indicates the better solubility of PCL in the respective solvent, which translates into stronger intermolecular interactions between polymer chains and solvent molecules. For problem-free ES and bead-free nanofibers, the choice of solvent and its solution viscosity plays a vital role. Therefore, a homogenous polymer solution with optimum viscosity is needed to perform successful ES processes. Higher solution viscosity and reduced surface tension contribute to the bead-free formation of nanofibers. The concentration of polymer controls the final solution-viscosity, and the coefficient of surface tension depends upon the interaction between polymer and solvent. For example, the addition of ethanol to the solvent system can reduce the surface tension coefficient, and this will avoid the bead formation and will ensure smooth ES of nanofibers under electric field [26]. Therefore, after analyzing these viscosity values, the binary solvent system of chloroform:ethanol (88:12 wt/wt) was selected as a suitable solvent for preparing PCL solutions. All the experiments in this study were performed using this binary solvent system.

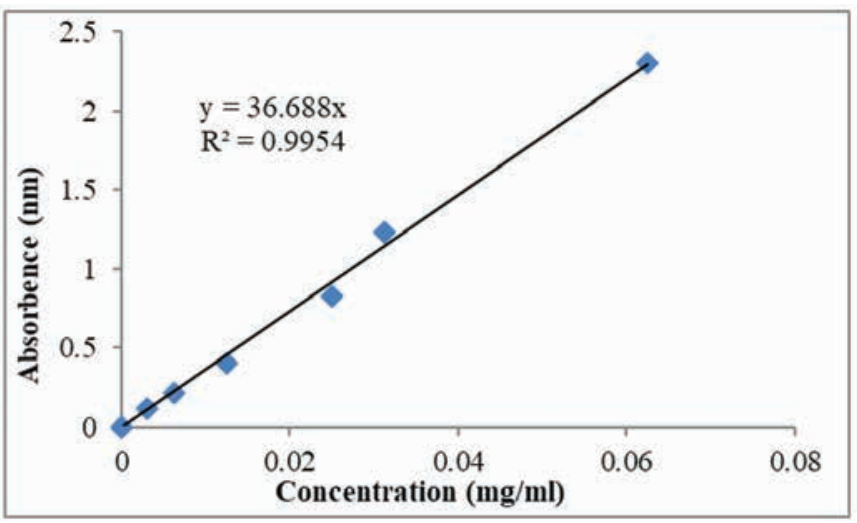

Figure 3. Calibration curve for IBU release. IBU, ibuprofen. 
Table 1. Viscosities of $10 \mathrm{wt} \% \mathrm{PCL}$ solutions in different solvents

\begin{tabular}{|c|c|}
\hline Solvent & Viscosity (MPa/s) \\
\hline Chloroform & 3,600 \\
\hline Chloroform: Ethanol (88:12 wt/wt) & 3,200 \\
\hline THF & 620 \\
\hline Acetone & 510 \\
\hline o-xylene & 330 \\
\hline
\end{tabular}

PCL, poly(E-caprolactone); THF, tetrahydrofuran.

\subsection{Morphology and diameters of nanofibers}

On the basis of preliminary studies of ES parameters and their impact on nanofibers' characteristics, the optimum ES parameters were selected. These optimum ES parameters, for all the samples, are mentioned in Table 2. These best parameters were selected on the basis of homogeneity of these nanofibers with a small coefficient of variation of their mean diameters. The temperature of ES chamber was $25 \pm 2{ }^{\circ} \mathrm{C}$ and the relative humidity was $35 \pm 4 \%$ for all the experiments.

\subsubsection{Impact of PEG-400 on morphology of PCL nanofibers}

The morphology of nanofibrous webs was studied by SEM analysis and their diameters were measured by using ImageJ software, which was used to take 50 measurements per specimen. The SEM images of PCL, PCL/PEG, and their IBUloaded nanofibers showed predominantly round morphology with varying thicknesses along their length, as shown in Figure 4. No bead formation was observed in any sample. All samples were heterogeneous in morphology including nano and micro fibers and showed variations in their mean diameters. The diameters of PCL nanofibers were ranging from $500 \mathrm{~nm}$ to $3.5 \mu \mathrm{m}$ whereas, in case of PCL/PEG-10\%, nanofibers' diameters ranged from $400 \mathrm{~nm}$ to $1.5 \mu \mathrm{m}$ [27]. It was obvious that the addition of PEG-400 noticeably reduced the diameters of nanofibers. Moreover, the morphology of PCL/PEG nanofibers was also different because numerous pores were seen on their surface that could be due to the phase separation of both polymers; such separation was considered to be an expected result according to previously reported studies [19].

\subsubsection{Impact of IBU on morphology of PCL and PCL/PEG nanofibers}

Nanofibrous webs loaded with IBU showed a homogenous morphology with larger diameters ranging from $2 \mu \mathrm{m}$ to $3 \mu \mathrm{m}$. Larger diameters due to IBU were in agreement with the fact that IBU impacted the nanofibers as a defect. However, IBUincorporated nanofibers, in presence of PEG-400, showed a decline in their diameters to a certain extent (ranging between $900 \mathrm{~nm}$ and $2.1 \mu \mathrm{m}$ ). This impact of PEG-400 was similar to the case of PCL/PEG-10\% nanofibers. The overall morphology of nanofibers was heterogeneous, with $\mathrm{CV} \%$ ranging from $10-40 \%$. In a few SEM images, agglomerates of IBU were observed on the surface of PCL and PCL/PEG nanofibers.

\subsection{Tailoring the wettability of PCL nanofibers with PEG- $\underline{400}$}

Despite its good mechanical properties, slow degradation and biocompatibility, PCL lacks hydrophilicity, and this lacuna limits its applicability for wound healing. Therefore, a hypothesis was made that blending of PCL with a hydrophilic polymer such as PEG-400 would solve this problem. This theory was verified by taking water contact angle measurements for pristine $\mathrm{PCL}$

Table 2. Optimum ES parameters for PCL, PCL/PEG, and their IBU loaded nanofibers

\begin{tabular}{|c|c|c|c|}
\hline Sample & Applied voltage (kV) & Needle-collector distance (cm) & Feed rate (mL/h) \\
\hline PCL & 25 & 25 & 0.5 \\
\hline PCL/PEG-10\% & 20 & 25 & 0.5 \\
\hline PCL/PEG-20\% & 20 & 25 & 0.5 \\
\hline 5 wt\%IBU+PCL & 20 & 25 & 0.5 \\
\hline 7 wt\%IBU+PCL/PEG-10\% & 20 & 25 & 0.5 \\
\hline
\end{tabular}

ES, electrospinning; IBU, ibuprofen; PCL, poly(E-caprolactone); PEG, poly(ethylene glycol).

Table 3. Results of water contact angle measurements for pure PCL film, PCL, and PCL/PEG-10\% nanofibrous webs

\begin{tabular}{|c|c|c|}
\hline Sample & Mean water contact angle & Observations \\
\hline Pure PCL film & $72^{\circ} \pm 1.2^{\circ}$ & Hydrophobic \\
\hline PCL nanofibers & $130^{\circ} \pm 1.9^{\circ}$ & Hydrophobic \\
\hline PCL/PEG-10\% nanofibers & †Rapid absorption & Hydrophilic \\
\hline
\end{tabular}

PCL, poly(E-caprolactone); PEG, poly(ethylene glycol).

tWater droplet was instantly absorbed due to the presence of PEG-400 on the surface of nanofibers. 

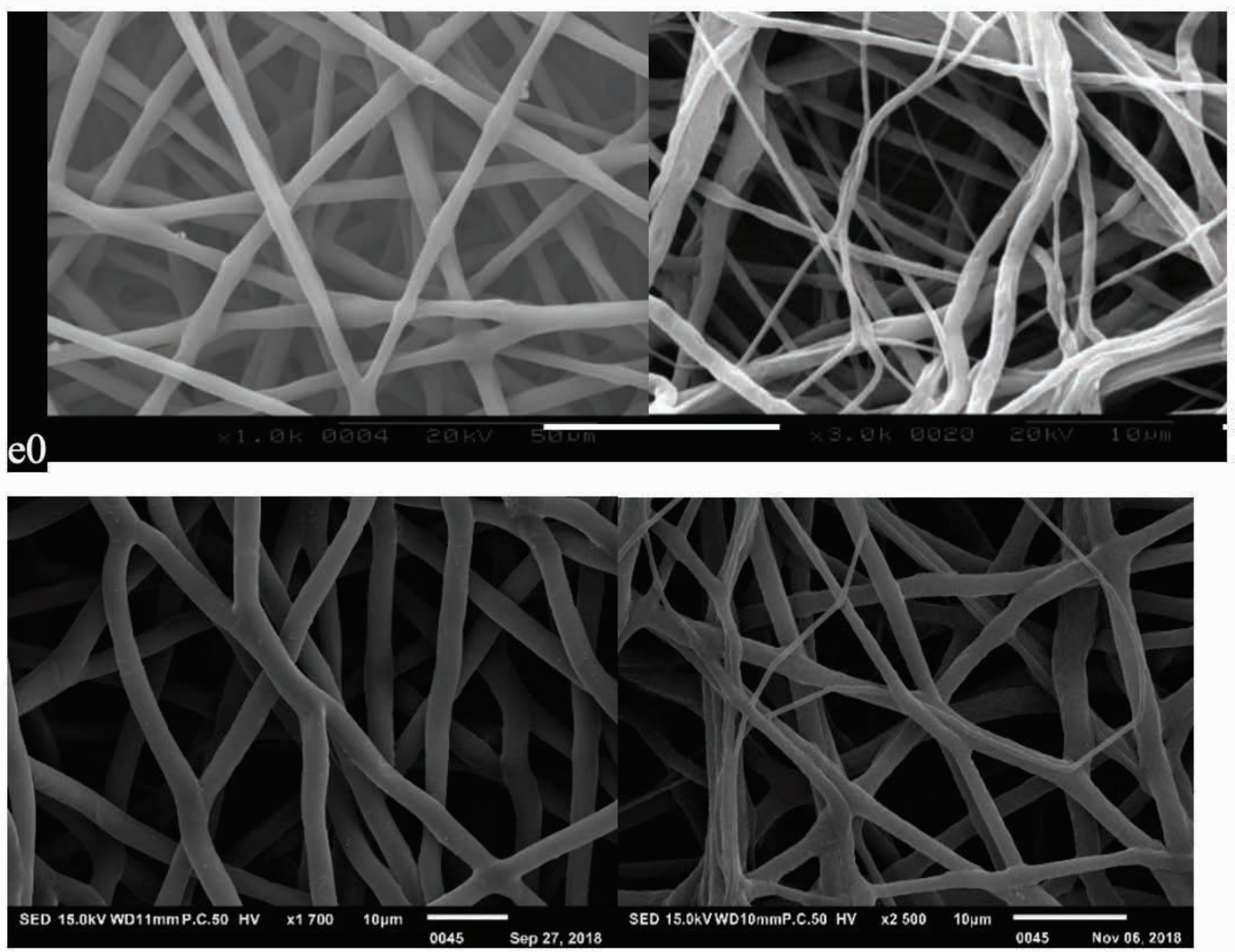

Figure 4. SEM micrographs of PCL, PCL/PEG-10\%, 5\%IBU + PCL, and 7\%IBU + PCL/PEG-10\% nanofibers. IBU, ibuprofen; PCL, poly $(\mathcal{E}$ caprolactone); PEG, poly(ethylene glycol); SEM, scanning electron microscopy.

film, PCL, and PCL/PEG-10\% nanofibrous webs, as given in Table 3.

It was observed that PCL nanofibers showed a larger water contact angle as compared to pure PCL film, as illustrated in Figure 5. A larger angle in case of PCL nanofibers could be due to the random arrangement of PCL chains in nanofibrous web structure, which resulted in a more compact surface and depicted enhanced hydrophobic behavior. The topology of these nanofibers plays an important role in determining their wettability. Surface roughness is known to amplify the hydrophobicity by mimicking the lotus effect [28]. Another reason for this phenomenon could be the stretching of $\mathrm{PCL}$ chains during ES, under the influence of electric fields that cause a rearrangement of polymer chains to form these nanofibers [29]. Contrastingly, in PCL/PEG-10\% nanofibers, it was not possible to measure the contact angle, since the water droplet was quickly absorbed on the surface of nanofibrous webs. This rapid absorption of the water droplet could be due to the presence of hydro soluble PEG-400 on the surface of nanofibers. Therefore, it was proved that the addition of PEG400 improved the wettability of PCL nanofibers, and this was actually a desired feature for its potential application as a wound dressing material to absorb wound exudate $[18,30]$.

\subsection{Crystallinity ratio of nanofibers}

\subsubsection{Impact of PEG-400}

DSC analysis was performed for pure PCL, PCL nanofibers, and PCL/PEG-10\% nanofibers to determine their glass transition temperatures $\left(T_{g}\right)$, melting temperatures $\left(T_{m}\right)$, and crystallinity ratios $\left(X_{c} \%\right)$. The results were analyzed by TA Universal Analysis and are shown in Table 4.

It was found that the crystallinity ratio of PCL nanofibers was $11 \%$ higher as compared to pure PCL $[24,31]$. This indicates that a change occurred in alignment of PCL chains during the ES process, that enhanced the overall crystallinity of $\mathrm{PCL}$ nanofibers. This is understandable, since the polymer solution underwent a stretching when it was subjected to a high voltage electric field that might have affected the crystallization phenomenon of polymer. This effect was more pronounced in nanofibers containing PEG-400 with crystallinity ratios up to $58 \%$. They exhibited an upsurge of $18 \%$, as compared to the crystallinity ratio of pure PCL that was $40 \%$. Another reason of this amplified crystallinity could be the PEG-400 polarity induced into the polymer solution that favored crystalline arrangement of macro molecules under the electric field. 


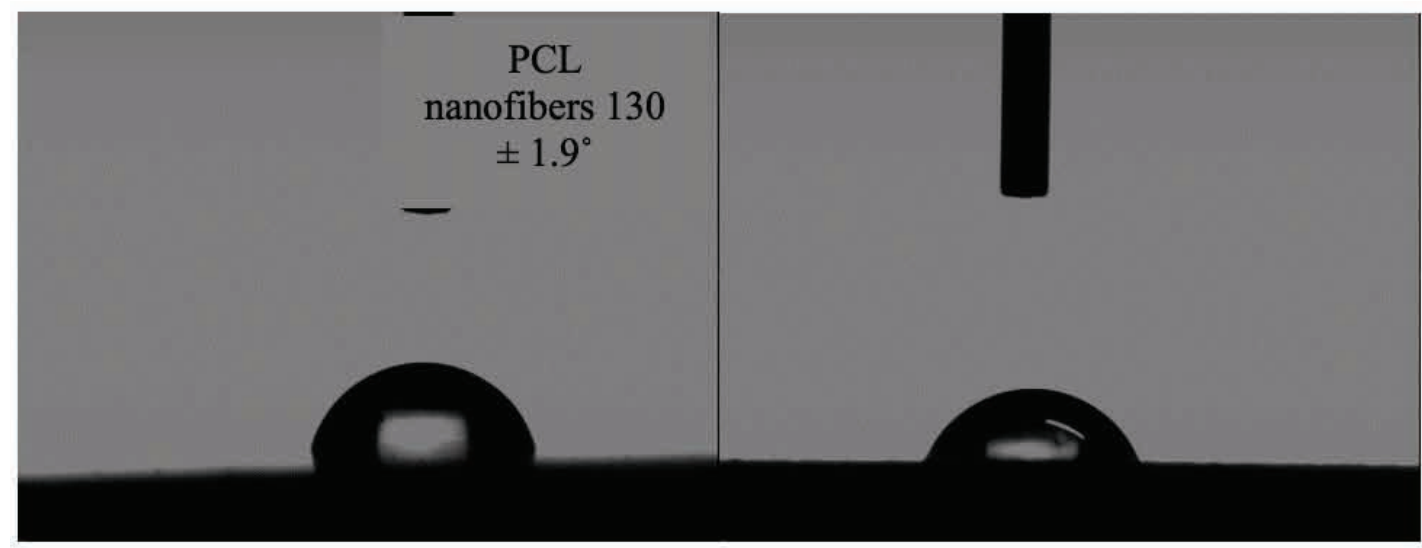

Figure 5. Water contact angles of PCL nanofibers and of pure PCL film. PCL, poly( $(\varepsilon$-caprolactone).

Table 4. Melting temperatures $\left(T_{m}\right)$, glass transition temperatures $\left(T_{g}\right)$, and crystallinity ratios $\left(X_{c} \%\right)$ of PCL, PCL/PEG-10\%, and IBU-loaded nanofibers determined by first heating cycle from $-80^{\circ} \mathrm{C}$ to $100^{\circ} \mathrm{C}$ under $\mathrm{N}_{2}$

\begin{tabular}{|c|c|c|c|c|}
\hline Sample composition & $\mathbf{T g}\left({ }^{\circ} \mathbf{C}\right)$ & $\mathbf{T m}\left({ }^{\circ} \mathbf{C}\right)$ & $\mathbf{D H m}(\mathbf{J} / \mathbf{g})$ & $\mathbf{X c}(\%)$ \\
\hline Pure PCL & -64 & 57 & 63 & 40 \\
\hline PCL nanofibers & -64 & 59 & 72 & 51 \\
\hline PCL+PEG-10\% nanofibers & -63 & 57 & 74 & 58 \\
\hline 5\% IBU+PCL nanofibers & -60 & 51 & 53 & 39 \\
\hline $7 \%$ IBU+PCL/PEG-10\% nanofibers & -61 & 50 & 59 & 46 \\
\hline
\end{tabular}

IBU, ibuprofen; PCL, poly(E-caprolactone); PEG, poly(ethylene glycol).

However, no significant change in glass transition and melting temperatures of nanofibers was recorded.

\subsubsection{Impact of IBU incorporation}

The DSC analysis of IBU-incorporated nanofibers was also done to investigate the effect of IBU on their crystallinity ratios. DSC analysis of $5 \% \mathrm{IBU}+\mathrm{PCL}$ and $7 \% \mathrm{IBU}+\mathrm{PCL} / \mathrm{PEG}-10 \%$ nanofibers showed moderate values of crystallinity with no sharp increase or decrease, as was the case in the PCL and PCL/PEG-10\% nanofibers. This implied that IBU acted as a defect in nanofibers' structure and hindered their crystalline arrangement. It can be said that in presence of $\mathrm{IBU}$, the $\mathrm{PCL}$ chains had less opportunity to rearrange, nucleate, and crystallize [32-34].

\subsection{Thermal stability of nanofibers}

The results of TGA analysis enabled comparison of the thermal properties of PCL, PCL/PEG-10\%, and their IBUloaded nanofibers with their pure states, as shown in Table 5. No residue was found at the end of the heating cycle for any sample. No significant difference among the degradation temperatures of PCL, of PCL/PEG-10\% nanofibers, and of pure PCL was observed, which implicates that addition of PEG400 did not affect the thermal stability of PCL nanofibers. The IBU-loaded nanofibers showed a decline in their degradation temperatures, and this degradation is in agreement with their decreased crystallinity ratios determined by DSC analysis. Despite the decrease in their degradation temperatures, IBU-

Table 5. TGA results of PCL, PCL/PEG-10\%, and IBU loaded nanofibers compared to their pure states for $800^{\circ} \mathrm{C}$ heating ramp under N2

\begin{tabular}{|c|c|c|}
\hline Sample composition & 5\% degrad. temp. $\left({ }^{\circ} \mathbf{C}\right)$ & Total degrad. temp. $\left({ }^{\circ} \mathbf{C}\right)$ \\
\hline Pure PCL & 365 & 230 \\
\hline Pure IBU & 159 & 375 \\
\hline Pure PEG & 245 & 580 \\
\hline 10 wt $\%$ PCL & 360 & 470 \\
\hline PCL/PEG-10\% & 302 & 429 \\
\hline $5 \%$ IBU + PCL Nanofibers & 208 & 353 \\
\hline
\end{tabular}

IBU, ibuprofen; PCL, poly(E-caprolactone); PEG, poly(ethylene glycol); TGA, thermogravimetric analysis. 
loaded nanofibers still exhibit adequate thermal stability for their potential application as wound-dressing material.

\subsubsection{Presence of IBU determined by TGA}

Thermal analysis of IBU-loaded nanofibers also confirmed the presence of IBU in the nanofibers' structure. The degradation curves of pure IBU, $5 \% \mathrm{IBU}+\mathrm{PCL}$, and $7 \% \mathrm{IBU}+\mathrm{PCL}-\mathrm{PEG}-10 \%$ nanofibers are shown in Figure $6(a, b$ and $c)$ respectively.

The degradation curve of pure IBU showed that a complete degradation of drug takes place at $226^{\circ} \mathrm{C}$, as shown in Figure 6(a). Therefore, similar intermediate peaks at $228^{\circ} \mathrm{C}$ and $230^{\circ} \mathrm{C}$ were noted in degradation curves of $5 \% \mathrm{IBU}+\mathrm{PCL}$ and $7 \%$ IBU + PCL/PEG-10\% nanofibers, respectively. Presence of these corresponding peaks of IBU during thermal degradation of these electrospun nanofibers provided strong evidence that the drug was successfully incorporated into the network of nanofibers during their ES.

\subsection{Mechanical properties of nanofibers}

Fundamental studies on mechanical properties of electrospun nanofibers typically focus on uniaxial tensile-strength testing of randomly aligned nanofibers. In this study, tensile-strength testing of PCL/PEG-10\% and 7\%IBU + PCL/PEG-10\% nanofibrous webs (electrospun with chloroform:ethanol (88:12 wt/wt) as solvent) were performed. These nanofibrous webs consisted of randomly oriented nanofibers having heterogeneous morphology with their mean diameters ranging from nano to micro scale. The stress-strain curves of these randomly oriented PCL/PEG-10\% and 7\%IBU + PCL/PEG$10 \%$ nanofibrous webs are shown in Figure 7 , which highlights their mechanical behavior. Five samples of each type of nanofibers were tested.

These curves can be divided into three sections i.e., elastic, yielding, and strain-hardening regions. Specifically, the initial linear elastic region occurs up to $2 \%$ strain, where stretching and alignment of nanofibrous mats occurred along the direction of applied load. The values of applied tensile stress corresponding to their percentage strain for each sample are given in Table 6.

It was observed that in PCL/PEG-10\% nanofibers, when the applied stress increased up to $5 \mathrm{MPa}$, the percentage strain in material was $710 \%$, with a total elongation in material up to $66 \mathrm{~mm}$. On the other hand, in IBU-loaded nanofibers, the value for maximum applied stress only reached up to $1.8 \mathrm{MPa}$ and the resulting strain was up to $380 \%$ with elongation at a
A

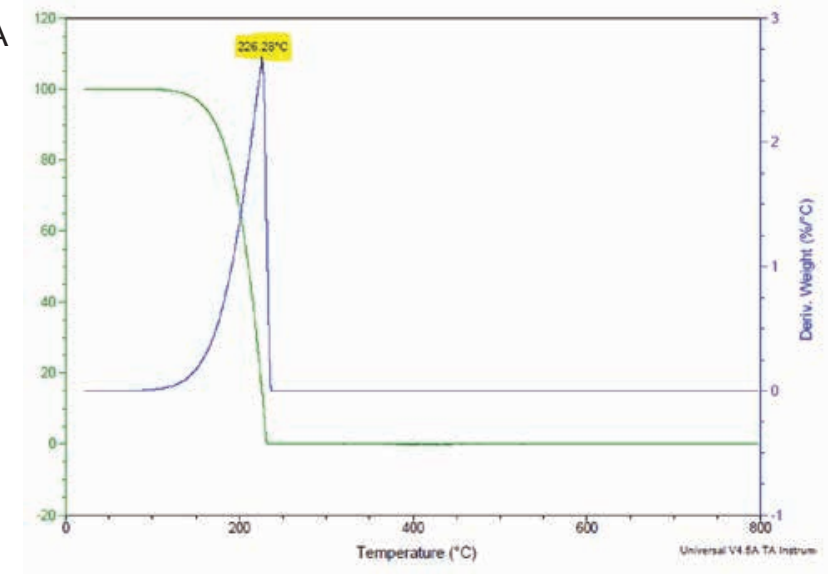

B
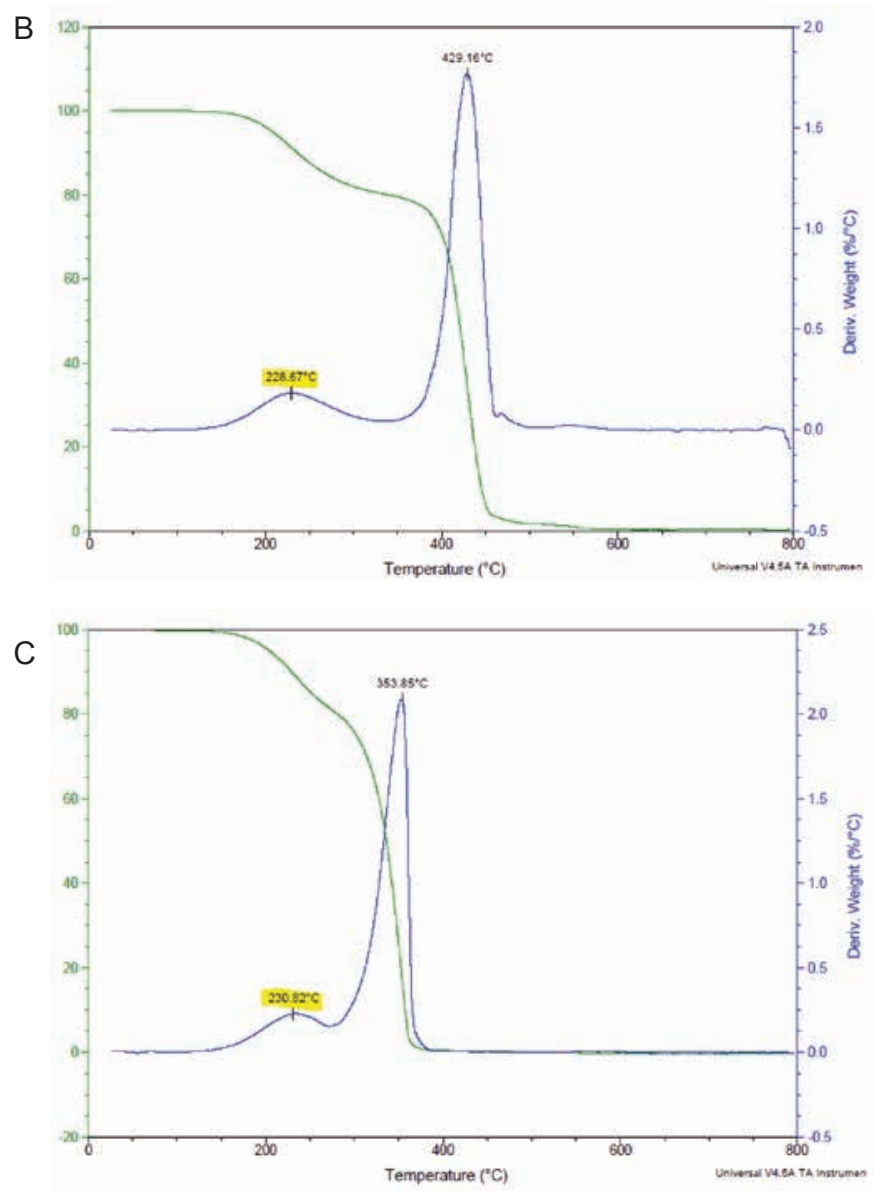

Figure 6. (a) Degradation curve for pure IBU at $10^{\circ} \mathrm{C} / \mathrm{min}$ heating rate till $800^{\circ} \mathrm{C}$ under $\mathrm{N}_{2}$. (b) Degradation curve for $5 \% \mathrm{IBU}+\mathrm{PCL}$ nanofibers at $10^{\circ} \mathrm{C} / \mathrm{min}$ heating rate till $800^{\circ} \mathrm{C}$ under $\mathrm{N}_{2}$. (c) Degradation curve for $7 \% \mathrm{IBU}+\mathrm{PCL} / \mathrm{PEG}-10 \%$ nanofibers at $10 \mathrm{C} / \mathrm{min}$ heating rate till $800 \mathrm{C}$ under $\mathrm{N}_{2}$. IBU ibuprofen; PCL, poly( $(\mathcal{\text { -caprolactone } ) ; ~ P E G , ~}$ poly(ethylene glycol).

Table 6. Maximum tensile stress and strain\% in PCL/PEG-10\% and 7\%IBU + PC/PEG-10\% nanofibers

\begin{tabular}{|c|c|c|c|}
\hline Sample & Nanofibers mean diameters (nm) & Stress (MPa) & Strain (\%) \\
\hline PCL/PEG-10\% & 1,200 & $5 \pm 0.7$ & $56 \%$ \\
\hline $7 \% \mathrm{IBU}+$ PCL/PEG-10\% & 2,100 & $1.8 \pm 0.25$ & $220 \%$ \\
\hline
\end{tabular}

IBU, ibuprofen; PCL, poly( $\varepsilon$-caprolactone); PEG, poly(ethylene glycol).

*Note: The original length of the nanofibers sample used for testing was $10 \mathrm{~mm}$. 

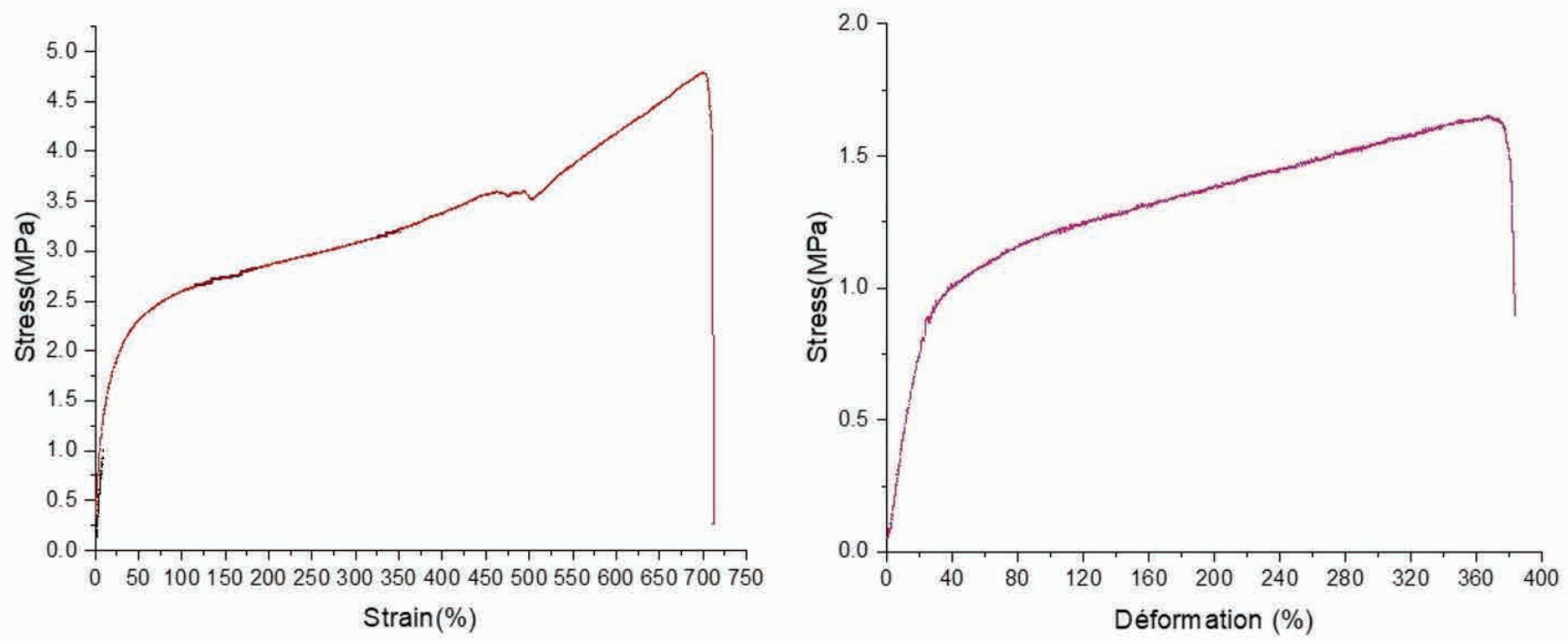

Figure 7. Stress-Strain curves of PCL/PEG-10\% and 7\%IBU + PCL/PEG-10\% nanofibers. IBU, ibuprofen; PCL, poly( $\mathcal{E}$-caprolactone); PEG, poly(ethylene glycol).

break of $32 \mathrm{~mm}$ in the material. The values for the tensile strength of IBU-incorporated nanofiber webs were lower than that of PCL/PEG-10\% nanofiber webs $[18,19]$.

\subsection{Drug release kinetics of IBU}

The main objective of drug incorporation into PCL and PCL/ PEG-10\% nanofibers was to devise a drug delivery system in these non-woven electrospun nanofibrous webs for their wound dressing applications. The performance of these IBUloaded nanofibrous webs was tested through in-vitro drugrelease kinetic study. Samples of these nanofibrous webs were immersed in phosphate-buffered saline (PBS) solution $(\mathrm{pH}=7)$ at $37^{\circ} \mathrm{C}$. Drug-release efficiency of IBU was monitored for a 24 h-duration. Percentage release efficiencies (RE\%) of both samples, 5\%IBU + PCL and $7 \mathrm{wt} \% \mathrm{IBU}+\mathrm{PCL} / \mathrm{PEG}-10 \%$, are given in Table 7 .

The graphical release profile of IBU in both samples is presented in Figure 8. The drug release analysis was performed for a 24 h-duration.

An initial burst release of IBU was observed during initial hours of analysis, followed by a gradual release until completion of $24 \mathrm{~h}$. This rapid release of IBU during the first $2 \mathrm{~h}$ could be attributed to the presence of IBU agglomerates on the surface of nanofibers. However, in case of $7 \mathrm{wt} \% \mathrm{IBU}+\mathrm{PCL} / \mathrm{PEG}-10 \%$,

Table 7. Initial and final release efficiencies (RE\%) of IBU from nanofibers

\begin{tabular}{|c|c|c|}
\hline Samples & RE\% (1.5 h) & RE\% (24 h) \\
\hline $5 \% \mathrm{IBU}+\mathrm{PCL}$ & $51 \%$ & $85 \%$ \\
\hline $7 \% \mathrm{IBU}+\mathrm{PCL} / \mathrm{PEG}-10 \%$ & $39 \%$ & $82 \%$ \\
\hline
\end{tabular}

ES, Electrspinning; IBU, ibuprofen; PCL, poly(E-caprolactone); PEG, poly(ethylene glycol).

${ }^{\dagger}$ ES conditions: $20 \mathrm{kV}$ applied voltage, $25 \mathrm{~cm}$ needle to collector distance, and $0.5 \mathrm{~mL} / \mathrm{h}$ feed rate.

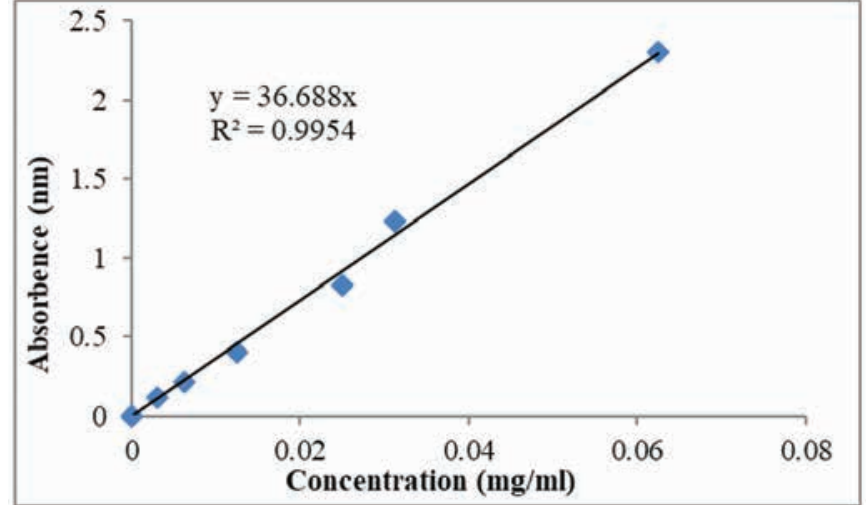

Figure 8. IBU-release profile from drug-loaded PCL and PCL/PEG$10 \%$ nanofibers. IBU, ibuprofen; PCL, poly $(\mathcal{E}$-caprolactone); PEG, poly(ethylene glycol).

this effect of burst release was rather slow as compared to the $5 \% \mathrm{IBU}+\mathrm{PCL}$ nanofibers [31-33]. This steady release could be due to the fact that IBU was embedded into the nanofibers' network and was not dispersed on the surface of nanofibrous webs. Furthermore, the amount of IBU released from these nanofibrous webs was not $100 \%$, which indicated that IBU was not homogenously disseminated into the nanofibrous network. Another possibility could be that some amount of IBU was lost during the ES process.

\section{CONCLUSIONS}

The ES and characterization of PCL and PCL/PEG-10\% nanofibers loaded with IBU were successfully executed. SEM analysis determined that nanofibers were round-shaped with heterogeneous morphology of diameters ranging from nano to micro scale. PCL/PEG-10\% nanofibers had pores on their surface due to the ejection of PEG-400 during ES. The addition of PEG-400 not only produced finer diameters but also enhanced the crystallinity ratio and wettability of $\mathrm{PCL}$ nanofibrous webs. Moreover, the incorporation of IBU yielded large diameters of nanofibers while having no substantial 
effect on their crystallinity ratio. PCL nanofibers showed good thermal stability and the presence of IBU was proved from its corresponding intermediate peaks that were found in the degradation curves of IBU-loaded nanofibers. It was established from the tensile strength analysis that IBU was responsible for a decline in tensile strength of PCL/PEG-10\% nanofibrous webs. The results of drug-release kinetics of IBUloaded nanofibers showed a burst release of IBU in the initial hours, followed by a gradual release during a 24 h-duration. The maximum IBU-release efficiencies recorded were up to $85 \%$ of the total amount of IBU incorporated. These results endorse the use of these IBU-loaded nanofibrous webs as a drug delivery system in biomedical wound dressings. These drug-incorporated nanofibrous webs can release IBU to relieve pain and inflammation and can absorb wound exudate to ensure rapid healing while protecting the wound from external environment. However, future efforts will be dedicated to achieving control over this burst release of drug and to devise a drug delivery system with steady prolonged release.

\section{Funding}

This work was supported by a grant of the Romanian Ministry of Education and Research, CNCS - UEFISCDI, project number PN-III-P1-1.1-TE-2019-0664, within PNCDI-III.

\section{Conflicting interests}

The authors declare that there exist no conflicts of interest.

\section{References}

[1] Sidgwick, G. P., McGeorge, D., Bayat, A. (2015). A comprehensive evidence-based review on the role of topicals and dressings in the management of skin scarring. Archives of Dermatological Research, 307, 461-477.

[2] Abrigo, M., McArthur, S. L., Kingshott, P. (2014). Electrospun nanofibers as dressings for chronic wound care: Advances, challenges and future prospects. Macromolecular Bioscience, 14, 772-792.

[3] Boateng, J. S., Matthews, K. H., Stevens, H. N. E., Eccleston, G. M. (2008). Wound healing dressings and drug delivery systems: A review. Journal of Pharmaceutical Sciences, 97, 2892-2923.

[4] Aragón, J., Costa, C., Coelhoso, I., Mendoza, G., AguiarRicardo, A., et al. (2019). Electrospun asymmetric membranes for wound dressing applications. Journal of Material Science \& Engineering C, 103, 109822.

[5] Bhardwaj, N., Kundu, S. C. (2010). Electrospinning: A fascinating fiber fabrication technique. Biotechnology Advances, 28, 325-347.

[6] Liao, N., Unnithan, A. R., Joshi, M. K., Tiwari, A. P., Hong, S. T., et al. (2015). Electrospun bioactive poly(Ecaprolactone)-cellulose acetate-dextran antibacterial composite mats for wound dressing applications. Colloids and Surfaces A, 469, 194-201.

[7] Pankongadisak, P., Sangklin, S., Chuysinuan, P., Suwantong, O., Supaphol, P. (2019). The use of electrospun curcumin-loaded poly(L-lactic acid) fiber mats as wound dressing materials. Journal of Drug Delivery Science and Technology, 536, 101-121.
[8] Goyal, R., Macri, L. K., Kaplan, H. M., Kohn, J. (2016). Nanoparticles and nanofibers for topical drug delivery. Journal of Controlled Release, 240, 77-92.

[9] Rodríguez-Tobías, H., Morales, G., Grande, D. (2019). Comprehensive review on electrospinning techniques as versatile approaches toward antimicrobial biopolymeric composite fibers. Material Science and Engineering C, 101, 306-322.

[10] Doustangi, A. (2015). Effect of electrospinning process parameters of polycaprolactone and nanohydroxyapatite nanocomposite nanofibers. Textile Research Journal, 85, 1445-1454.

[11] Beachley, V., Wen, X. (2009). Effect of electrospinning parameters on the nanofiber diameter and length. Material Science and Engineering C, 29, 663-668.

[12] Li, W., Shi, I., Zhang, X., Liu, K., Ismat Ullah, Cheng, P. (2018). Journal of Applied Polymer Science, 135.

[13] Huang, Z. M., Zhang, Y. Z., Kotaki, M., Ramakrishn, S. (2003). A review on polymer nanofibers by electrospinning and their applications in nanocpomposites. Composites Science and Technology, 63, 2223-2253.

[14] Dash, T. K., Konkimalla, V. B. (2012). Poly-e-caprolactone based formulations for drug delivery and tissue engineering: A review. Journal of Controlled Release, 158, 15-33.

[15] Woodruff, M. A., Hutmacher, D. (2010). The return of a forgotten polymer-polycaprolactone in the 21st century. Progress in Polymer Science, 35, 1217-1256.

[16] Elnakady, Y. A., AlRez, M. F., Fouad, H., Abuelreich, S., Albarrag, A. M., et al. (2015). Vascular tissue engineering using polycaprolactone nanofibrous scaffolds fabricated via electrospinning. Science of Advanced Materials, 7, 407-413.

[17] Detta, N., Brown, T. D., Edin, F. K., Albrecht, K., Chiellini, E., et al. (2010). Melt electrospinning of polycaprolactone and its blends with poly(ethylene glycol). Polymer International, 59, 1558-1562.

[18] Chena, C. H., Chen, S. H., Shalumona, K. T., Chena, J. P. (2015). Prevention of peritendinous adhesions with electrospun polyethylene glycol/polycaprolactone nanofibrous membranes. Colloids and Surfaces $B$ : Biointerfaces, 133, 221-230.

[19] Bui, H. T., Chung, O. H., Cruz, J. D., Park, J. S. (2014). Fabrication and characterization of electrospun curcuminloaded polycaprolactone-polyethylene glycol nanofibers for enhanced wound healing. Macromolecular Research, 22, 1288-1296.

[20] Repanas, A., Wolkers, W. F., Gryshkov O., Müller, M., Glasmacher, B. (2015). PCL/PEG electrospun fibers as drug carriers for the controlled delivery of dipyridamole. Journal of In Silico \& In Vitro Pharmacology, 1, 1-10.

[21] lurea, D. M., Popa, M., Chailan, J. F., Tamba, B. I., Todorancea, l., et al. Journal of Bioactive and Compatible Polymers, 28, 368-384.

[22] Li, J., Kuang, Y., Shi, J., Gao, Y., Zhou, J., et al. (2013). D-amino acids boost the selectivity and confer supramolecular hydrogels of a nonsteroidal antiinflammatory drug (NSAID). The Journal of Organic Chemistry, 135, 542-545.

[23] Li, F., Zhao, Y., Song, Y., Kumar, A. (2010). Nanofibers. IntechOpen. 
[24] Kolbu, D., Guimond-Lischer, S., Sajkiewicz, P., ManiuraWeber K., Fortunato G. (2015). The effect of selected electrospinning parameters on molecular structure of polycaprolactone nanofibers. International Journal of Polymeric Materials and Polymeric Biomaterials, 64, 365377.

[25] Qin, X., Wu, D. (2010). Effect of different solvents on poly(caprolactone) (PCL) electrospun nonwoven membranes. Journal of Thermal Analysis and Calorimetry, 107, 1007-1013.

[26] Angammana, C. J., Jayaram, S. H. (2011). Analysis of the effects of solution conductivity on electrospinning process and fiber morphology. IEEE Transactions on Industry Applications, 47, 1109-1117.

[27] Ferreira, J. L., Gomes, S., Henriques, C., Borges, J. P., Silva, J. C. (2014). Electrospinning polycaprolactone dissolved in glacial acetic acid. Journal of Applied Polymer Science, 131.

[28] Patankar, N. A. (2004). Mimicking the lotus effect: Influence of double roughness structures and slender pillars. ACS Publications, 20, 8209-8213.

[29] Tiwari, A. P., Joshi, M. K., Lee, J., Maharjan, B., Ko, S. W., et al. (2017). Colloids and Surfaces A: Physiochemical and Engineering Aspects, 520, 105-113.
[30] Guan, J., Wagner, W. R. (2005). Synthesis, characterization and cytocompatibility of polyurethaneurea elastomers with designed elastase sensitivity. Biomacromolecules, 6 , 2833-2842.

[31] Wang, X., Zhao, H., Turng, L. S., Li, Q. (2013). Crystalline morphology of electrospun poly(E-caprolactone) (PCL) nanofibers. Journal of Industrial Engineering Chemistry Research, 52, 4939-4949.

[32] Shi, Y., Wei, Z., Zhao, H., Liu, T., Dong, A., et al. (2013). Electrospinning of ibuprofen-loaded composite nanofibers for improving the performances of transdermal patches. Journal of Nanoscience and Nanotechnology, 13, 38553863.

[33] Karavasili, C., Bouropoulos, N., Kontopoulou, I., Smith, A., Merwe, S. M., etal. (2014). Preparation and characterization of multiactive electrospun fibers: poly-carpolactone fibers loaded with hydroxyapatite and selected NSAIDs. Journal of Biomedical Materials Research, 102, 2583-2589.

[34] Potrc, T., Baumgartner, S., Roskar, R., Planinsek, O., Lavric, Z., et al. (2015). Electrospun polycaprolactone nanofibers as a potential oromucosal delivery system for poorly water-soluble drugs. European Journal of Pharmaceutical Sciences, 75, 101-113. 